\title{
Perturbation Dynamics in Turbulent Flames
}

\author{
Malik Hassanaly*, Venkat Raman ${ }^{\dagger}$ \\ Department of Aerospace Engineering, University of Michigan, Ann Arbor, MI 48109-2102, USA
}

\begin{abstract}
A new approach based on dynamical systems theory is introduced in order to study turbulent combustion. Here, instead of the conventional statistical approach to chaotic systems, a perturbation-based dynamical systems approach is used. The main focus is in the characterization of the attractor, which represents the loci of solutions given a set of initial, boundary and operating conditions. In this paper, we discuss the basic formulation, the definition of so-called Lyapunov vectors and exponents, and the numerical approach for computing these quantities. The method is applied to a canonical premixed turbulent flame in a periodic box. The algorithms are developed in the context of low-Mach number direct numerical simulation solvers, which introduces certain unique complications. Tests of numerical convergence and behavior of the Lyapunov quantities are discussed.
\end{abstract}

\section{Introduction}

The description of turbulent combustion has long relied on a statistical approach, which utilized moments of an underlying distribution function to capture the main features of turbulence. In order to develop computational models, this method requires additional assumptions, which are typically of the form proposed by Kolmogorov. ${ }^{1,2}$ This leads to the analysis of turbulent flows in terms of length and time scales, and correlations that determine the interaction of these scales. Central to this approach to turbulence is the notion of equilibrium, which is often hidden in the models and theories, and almost never explicitly stated. For instance, the celebrated Kolmogorov's view of turbulence cascade is valid only when an equilibrium transfer of energy between the large and small scales is established. Turbulent combustion, which typically involves fast chemical reactions that should disrupt the isotropic nature of Kolmogorov turbulence, and provide energy to the small turbulent length scales, is still subject to the same treatment. Surprisingly, even this simplification still leads to useful computational models that have been widely validated using laboratory scale flames. ${ }^{3}$

Additional assumptions embedded in the usual treatment of turbulent combustion are questionable for predictive applications. Without considering modeling uncertainties from the transport equations, the deterministic approach for advancing flow fields in time requires a full knowledge of initial conditions. However for practical applications, only a partial and uncertain description of operational conditions can be reasonably obtained. The simulation outcome can then be described using random variables for which useful statistics can be obtained with classical approaches. Here, we seek a new approach which is geared towards specific realizations rather than statistical properties. We believe that this viewpoint will allow simulation of extreme or low-probability events which can lead to consequential, and often catastrophic, failure scenarios. For this purpose, a new computational tool is introduced to obtain information about individual outcome realizations rather than their statistics.

Consider the propagation of turbulent flame in a channel. ${ }^{4}$ A key quantity of interest is the location of the flame at some future time. Due to the chaotic nature of turbulent flow, given an incomplete set of initial conditions, many different locations are feasible. A computational model based on statistical theory can only determine (at best) the average location of the flame at a future time. But from a design perspective, it would be useful to have information on the distribution of flame locations. While it seems that this is a

${ }^{*} \mathrm{PhD}$ Student, University of Michigan, AIAA Student Member.

$\dagger$ Associate Professor, University of Michigan, AIAA Associate Fellow,

Copyright (c) 2016 by Malik Hassanaly. Published by the American Institute of Aeronautics and Astronautics, Inc. with permission. 
trivial issue (why not just repeat many 'slightly' different initial conditions and get this distribution?), the analysis shown below will demonstrate that this is a daunting task. In particular, the theory to efficiently perturb the initial conditions needs to be developed.

In this regard, the focus of this work is the development of a dynamical systems perspective of turbulent combustion, by relying on Lyapunov theory for the classification of turbulent processes. The study of turbulence through dynamical systems was popular in the mid 1980's and early 1990's, when algorithms for computing Lyapunov spectrum became more readily available. ${ }^{5-8}$ However, the focus there was on representing the complex dynamical process in terms of a lower-dimensional system (model reduction). These efforts quickly faded with the recognition that the number of active dimensions scales super-linearly with Reynolds number. ${ }^{9}$ Consequently, the dynamical system will be vastly complex for high Reynolds number flows. Instead, with a limited description of the spectrum, it was used to understand perturbation growth mechanism in turbulent application. ${ }^{10,11}$

Although computationally intensive, we believe that the notion of Lyapunov-theory based description of turbulence is powerful. The use of Lyapunov theory is more practical now due to three different reasons: 1) Computers have become significantly more powerful, with algorithms for specific linear algebra operations more efficient than before, 2) the use of Lyapunov theory has progressed in the field of atmospheric weather prediction, leading to novel insights into the significance and utility of this approach, ${ }^{12}$ and 3 ) turbulent combustion systems contain non-negligible volume of low-Reynolds number flow in order to stabilize the flame. Such regions ultimately determine the departure from average behavior. It is our belief then that these systems are more amenable to Lyapunov theory.

With this background, the objective in this paper is to introduce the Lyapunov exponents and vectors for turbulent combustion applications, provide algorithms for determination of these quantities, and lay the groundwork for simulating low-probability dynamical events in realistic flow problems.

\section{Dynamical systems representation of governing equations}

In the dynamical systems perspective, the partial differential equations (PDE) governing the fluid flow and combustion are converted into a finite-dimensional set of ordinary differential equations (ODE). This is equivalent to assuming a sufficiently fine grid, and discretizing the equations. The resulting set of ODEs can be represented as

$$
\frac{d \mathbf{X}}{d t}=\mathcal{F}(\mathbf{X}) ; \mathbf{X}(t=0)=\mathbf{X}^{0},
$$

where $\mathbf{X}$ is the vector of variables, and $\mathcal{F}$ is the discretized governing equations. In terms of PDE, if a computational grid with $N$ control volumes/grid points is used, and $S$ equations are solved, the length of the corresponding $\mathbf{X}$ vector will be $\mathcal{D}=S \times N$, which is the dimension of the state space for this system. The state space is also called solution space or phase space in dynamical systems theory. These three terms will be interchangeably used in the rest of the paper. The state space is high-dimensional and will be described using a linear combination of spanning vectors. These vectors will be called state vectors or solution vectors in the rest of the paper.

The main procedure is to determine the response of a turbulent combustion system to a perturbation. Using the aforementioned notations, one can consider a variation (perturbation) in state-space, given by $\delta \mathbf{X}$, which will evolve according to

$$
\frac{d \delta \mathbf{X}}{d t}=\frac{\partial \mathcal{F}}{\partial \mathbf{X}} \delta \mathbf{X}=\mathcal{J}(\mathbf{X}) \delta \mathbf{X} ; \quad \delta \mathbf{X}\left(t=T_{0}\right)=\delta \mathbf{X}^{0},
$$

This equation can be integrated in time along with the governing equation (Eq. 1) to obtain the following at time $T$

$$
\delta \mathbf{X}(T)=\delta \mathbf{X}\left(T_{0}\right) \int_{T_{0}}^{T} \mathcal{J}(\mathbf{X}) d t,
$$

where the term inside the integral is independent of the perturbation. Based on this relation, an expansion rate can be expressed as

$$
\Lambda=\frac{1}{T-T_{0}} \log \frac{\|\mathbf{X}(T)\|}{\left\|\mathbf{X}\left(T_{0}\right)\right\|} .
$$

This quantity $\Lambda$ determines the rate at which an initial change to the flow field grows with time, between $T$ and $T_{0}$. If $\Lambda$ is negative, the system returns to the original state after a finite time (determined by the 
value of the expansion rate). In general, $\Lambda$ is a function of the local phase space (and therefore of the time) at which it was determined and $\Lambda$ is allowed to oscillate between negative and positive values as the phase space evolves. It is then possible to extract a mean value for the expansion rates

$$
\lambda \equiv \lim _{T \rightarrow \infty} \frac{1}{T} \int_{0}^{T} \Lambda(t) d t
$$

Since the original solution spans an $\mathcal{D}$-dimensional space, it is possible to obtain expansion rates for each of these dimensions by considering $\mathcal{D}$ such perturbations applied to the original system. The procedure for obtaining these expansion rates became available in mid 1980s due to Benettin, ${ }^{5,13}$ which was then used to study a number of dynamical systems. ${ }^{6,8,10,12}$ Using this procedure, it is then possible to obtain a spectrum characterizing the dynamical response of the system. This Lyapunov spectrum consists of a set of time dependent vectors associated with mean expansion rates. These are computed using a Gram-Schmidt process. ${ }^{5,13}$ In general, the number of Lyapunov vectors and exponents equal the number of dimensions of the state-space. However, the procedure allows the selection of the first $N_{s}$ such vectors/exponents to be calculated, which ensures computational tractability.

The decomposition of the solution space in a hierarchy of vectors (vectors weighted with their associated mean Lyapunov exponent) is critical for the simulation of dynamical events. For a chaotic system, a subset of the LEs will be positive. The positive exponents determine the divergence of trajectories of nearby points in state-space at some initial time. The negative exponents, on the other hand, represent the convergence of trajectories. Hence, for any dynamical system, there exists a lower dimensional sub-space or volume that represents the dynamical motion of the system. This lower dimensional subspace is termed as an attractor (a strange attractor, as naming conventions go). The dimensionality of this attractor is obtained from the Kaplan-Yorke estimator. ${ }^{14}$

$$
\mathcal{N}_{D}=j+\frac{\sum_{l=1}^{j} \lambda_{l}}{\left|\lambda_{j+1}\right|} \sum_{l=1}^{j} \lambda_{l}>0 \text { and } \sum_{l}^{j+1} \lambda_{l}<0 .
$$

In general, the attractor dimension scales with the number of active modes in the dynamical system. In the case of turbulence, this would imply that an increase in Reynolds number will increase the dimensionality. Theoretical derivations have shown that the scale up can vary from the traditional Kolmogorov estimate of $\mathrm{Re}^{9 / 4}{ }^{9}$ However, due to the low Reynolds number stabilization region in combustion systems, this exponent is expected to be lower. Furthermore, the knowledge of the structure of the attractor provides critical insight about the direction in which the system can positively (amplify) or negatively (damp) perturbations applied to the solution.

With these objectives in mind, the rest of the paper is laid out as follows. The definition of the configuration in terms of a dynamical system is described in details in Sec. III. Numerical choices for the computation of the Lyapunov spectrum are discussed in Sec. IV. Finally, we comment on characteristic features found for the Lyapunov spectrum of the considered turbulent combustion configuration in Sec. V.

\section{Computation of Lyapunov exponents for a canonical premixed flame}

\section{A. Stationary premixed turbulent flame in a periodic box}

The configuration adopted for the present case is a freely propagating premixed flame in an open domain $2 \mathrm{~cm} \times 1 \mathrm{~cm} \times 1 \mathrm{~cm}$ (Fig. 1). Periodic boundary conditions are enforced normal to the $\mathrm{y}$ and $\mathrm{z}$ planes. A turbulent inflow is enforced at the inlet x-plane and convective outflow boundary conditions are applied at the outlet x-plane for both velocity and scalars.

The flame considered is a premixed $\mathrm{CH}_{4}$ flame, preheated to $750 \mathrm{~K}$ and at an equivalence ratio $\phi=1$. It is initialized as a flat plane at $x=1 \mathrm{~cm}$. The flame is modeled using tabulated chemistry obtained from a 1D unstretched premixed freely propagating flame obtained with Flamemaster. ${ }^{15}$ To reduce the state-space dimensionality, a progress variable $C$ is transported to represent the advancement of the reaction and is defined as

$$
C=\frac{Y_{C O_{2}}-Y_{C_{2}, u}}{Y_{C_{2}, b}-Y_{C O_{2}, u}}
$$

where $\mathrm{Y}_{\mathrm{CO}_{2}}$ is the local mass fraction of $\mathrm{CO}_{2}, Y_{\mathrm{CO}_{2}, u}$ is the mass fraction of $\mathrm{CO}_{2}$ at the unburnt boundary and $\mathrm{Y}_{\mathrm{CO}_{2}, b}$ is the mass fraction of $\mathrm{CO}_{2}$ at the burnt boundary. The tabulated mapping between the constructed 


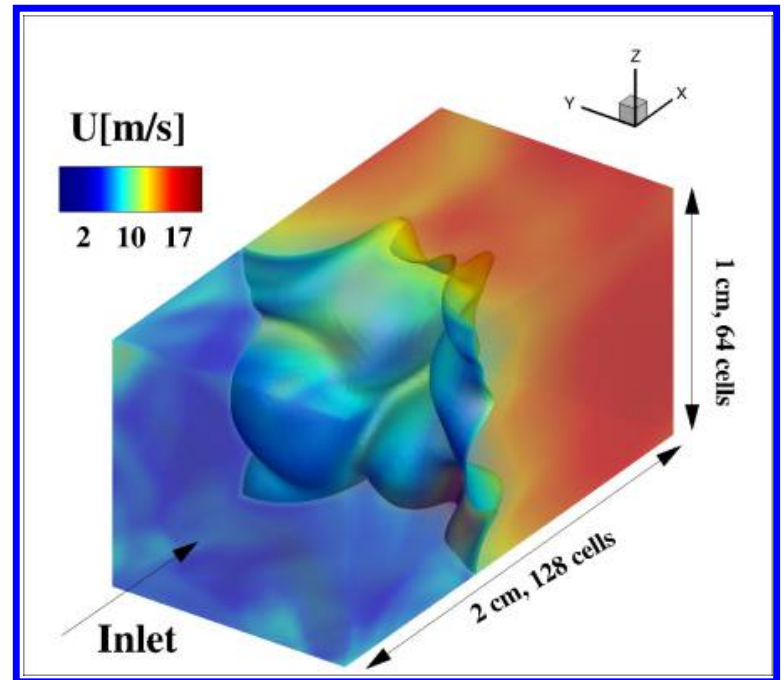

Figure 1: Contour of the streamwise velocity field superimposed with an isocontour of density $0.2 \mathrm{~kg} / \mathrm{m}^{3}$ identifying the flame front.

progress variable, temperature and progress variable source term is shown in Fig. 2. This should be considered equivalent to a 1-step chemistry model.

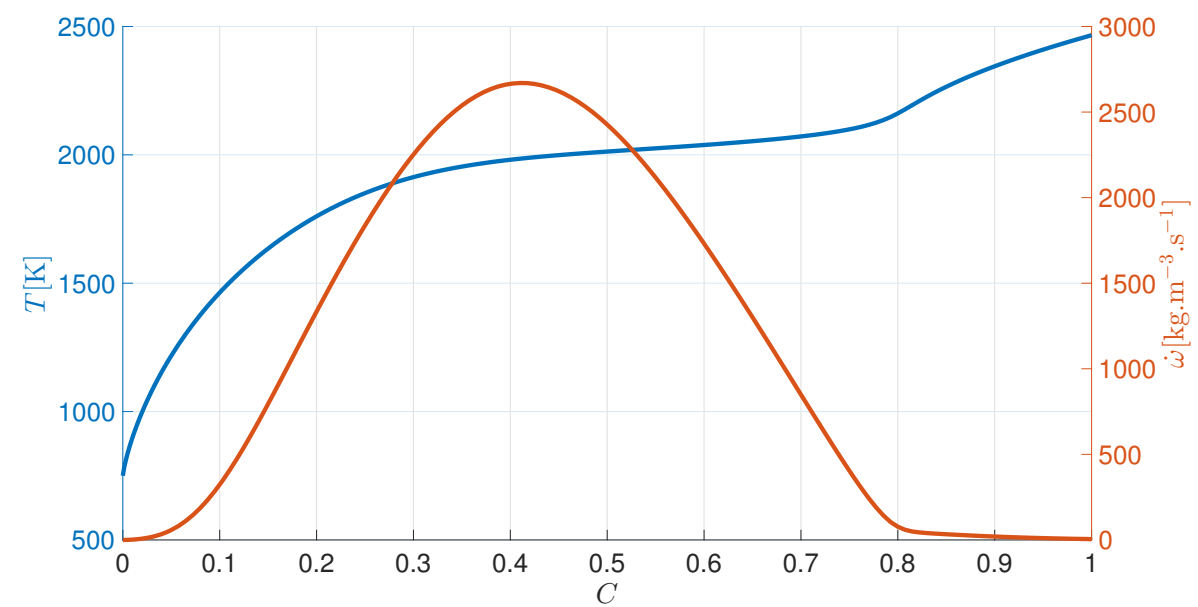

Figure 2: Tabulated mapping between the progress variable $C$ and the Temperature $T$ and progress variable source term $\dot{\omega}$

The turbulent velocity plane enforced at the inlet is obtained from an auxiliary homogeneous isotropic turbulence simulation (HIT) with the dimensions of the inlet plane. A linear forcing is applied to the stabilize the turbulence in the auxiliary HIT simulation, ${ }^{16}$ resulting in an eddy turnover time $\tau=1.34 e^{-4} s$ and an isotropic root mean square (RMS) velocity $u_{R M S}=1.2 \mathrm{~m} / \mathrm{s}$.

The premixed flame will propagate upstream or downstream based on the difference between the local flame front velocity and gas velocity. In order to stabilize the flame location, a control algorithm ${ }^{17}$ is utilized. Here, the bulk inflow velocity is modified in order to preserve the plane-averaged location of the flame front. Numerical details about the implementation of the control algorithm can be found in. ${ }^{18}$ The stabilization process is designed such that the flame comes back to its original position after 40 timesteps $(K=40$ using the notations of ${ }^{18}$ ). If the flame were not stabilized, it could leave the domain before meaningful statistics about the expansion rates are acquired.

It should also be noted that to dynamically stabilize the flame without modifying the turbulence level to which the flame is exposed, one should provide an inlet profile containing the physical streamwise dimensions of the auxiliary HIT simulation. With this method, as the bulk flow velocity pushes or pulls the flame, the size of the vortices issued from the inlet can be held constant. A straightforward approach is to use a 3D 
HIT field as the inlet file. The inlet bulk velocity then determines which streamwise slice of the inlet profile should be read.

The grid size is set to $128 \times 64 \times 64$ in the streamwise, spanwise, and stream-normal directions, respectively. A second-order finite volume method implemented in the low-Mach number code $\mathrm{NGA}^{19}$ is used. Due to the necessity of resolving the propagation of perturbations in a short time-scale, the timestep is set to $2.5 \mu \mathrm{s}$ resulting in a maximal CFL (Courant-Friedrichs-Lewy) number of about 0.3. Therefore, an explicit temporal scheme is used for the computations discussed below.

\section{B. Definition of state-space}

The state-space is defined by considering Eq 1: $\mathbf{X}$ should contain all the necessary information such that the evolution of the vector $\mathbf{X}$ is fully determined by itself. In other words, a dimension is added in the solution vector (or state-space) when it is not possible to determine its local values based on other solution vector fields. This would imply that certain fields, such as viscosity, are not independent fields since this value is determined directly from the flamelet table using the local progress variable value. Consequently, the state-space is modified to include only the truly independent variables

Furthermore, the development of the exponents should take into account the solution procedure for the forward problem (i.e, the solution approach to Eq. 1). In this study, a variable density low-Mach number solver is used. The velocity, the mass flux and the density are three different fields satisfying different constraints. The velocity field satisfies momentum conservation, the mass flux satisfies mass conservation, subject to density change for an exothermic combustion process.

Four fields are being transported: first the three momentum components using Eq. 8

$$
\frac{\partial \rho \mathbf{u}}{\partial t}+\nabla \cdot(\rho \mathbf{u u})=-\nabla p+\nabla \cdot\left(2 \mu\left(\mathbf{S}-\frac{1}{3} \mathbf{I} \nabla \cdot \mathbf{u}\right)\right)
$$

where $\mathbf{I}$ is the Kronecker delta vector, and $\mathbf{S}$ is the strain-rate tensor, and $\mu$ is the dynamic viscosity. The progress variable is transported using Eq. 9

$$
\frac{\partial \rho C}{\partial t}+\nabla \cdot(\rho \mathbf{u} C)=\nabla(D \nabla C)+\dot{\omega}
$$

where $D$ is the mass diffusivity of the progress variable and $\dot{\omega}$ is the progress variable source term due to the combustion process.

For the canonical case shown in this study, the use of a premixed flamelet method allows to directly correlate the viscosity $\mu$, the diffusivity $D$, the density $\rho$ and the reaction source term $\dot{\omega}$ to the transported progress variable $C$. It should be noted that a direct correspondence between $C$ and $\rho$ is enforced, which may not exactly conserve $\rho C$, but is not critical to the present discussion. Thus $C$ is included in the state-vector because of the particular choice of the combustion model, and its link to the density field. The pressure field is included because the first outer-iteration of the solver uses the previous pressure field as a guess for the next pressure field. The iterative procedure for the momentum equation can be written as follows:

$$
\left(\text { Time }^{*}\right)_{k}^{n+1}+(\text { Conv })_{k}^{n+1}=(\text { Press })_{k-1}^{n+1}+(\text { Visc })_{k}^{n+1} \text { and }(\text { Time })_{k}^{n+1}=\left(\text { Time }^{*}\right)_{k}^{n+1}+(\text { Press })_{k}^{n+1}
$$

where the superscript is the timestep index, and the subscript is the outer-iteration index. (Time) is the time derivative term in the momentum equation, (Conv) is the convection term, $(\mathrm{Visc})$, is the viscous term, $(\text { Press })_{k-1}^{n+1}$ is a first guess for the pressure term and (Press $)_{k}^{n+1}$ is a correction pressure term obtained from a Poisson equation. At the first outer-iteration $(k=1)$ the first guess for pressure is written as $(\text { Press })_{3}^{n}$ which then requires to use pressure in the state vector.

Because at the beginning of every timestep $(n+1)$, the previous timestep fields $(n)$ are initialized using the current values available, there is no need to carry old fields. In particular, the variation of density $\frac{\delta \rho}{\delta t}$ (where $\delta$ denotes some discretization scheme), does not need to be stored in the solution vector. With no modifications added to the NGA-code, the dynamical system can a priori be written as a solution vector containing 8 variables $\mathbf{X}=\{U, V, W, \rho U, \rho V, \rho W, C, P\}$ where $U, V, W$ (respectively $\rho U, \rho V, \rho W$ ) are the velocities (respectively mass fluxes) in the x,y and z-directions. Since $\mathbf{X}\left(t=t_{0}\right)$ fully determines $\mathbf{X}\left(t=t_{0}+\Delta t\right)$, it also determines $\frac{d \mathbf{X}}{d t}$. It will be seen in Sec.IV that useful reduction of the state space are possible with code modifications. 


\section{Numerical procedure}

With the current definition of the state space (Sec. B), the numerical method introduced by Benettin et al. ${ }^{5}$ can be used. Some numerical details not mentioned in their paper are explored in Sec. A. Details about the implementation are also provided in this section. The choice of a reduced solution vector is justified in Sec. D. Finally, a set of parameters necessary to conduct the Lyapunov spectrum calculation is determined using a convergence study.

\section{A. Revisited numerical procedure}

Benettin et al. ${ }^{5}$ introduced a numerical method to compute all the Lyapunov exponents of a dynamical system (also called cocycle). In this method, in order to compute the first $M$ Lyapunov exponents, it is required to evolve $M$ non-linearly dependent perturbations $\delta \mathbf{X}_{i}, i=1, \cdots, M$. The initial conditions are chosen such that this set of $M$ vectors forms an orthonormal basis of a $M$-dimensional subspace of the phase space. In practice $M+1$ simulations are performed: one reference simulation and $M$ perturbed simulations. The perturbations are propagated for a finite time, after which the expansion rates are computed. This finitetime is expressed as a number $s$ of iterations in. ${ }^{5}$ After $s$ iterations, the initially orthonormal perturbations are no longer orthonormal. In fact they tend to all align along the most expanding vector which can create numerical problems to determine other growing directions. To ensure numerical accuracy, the vectors are re-orthonormalized using a Gram-Schmidt process. This procedure is continued until the expansion rates are converged. The globally converged Lyapunov exponents are then obtained from the expansion rates in successive sub-volumes based on the multiplicative theorem of Oseledec. ${ }^{5}$ The vectors obtained are oriented in arbitrary directions but provide a description of the most expanding subspaces of the system.

Hidden in this description, two parameters need to be determined: the initial perturbation norm and the expansion rate averaging time. A third parameter not mentioned in the paper is the transient dissipation time after each modification of the perturbation. This parameter was not discussed by Benettin et al. ${ }^{5}$ because the problems they tackled were weakly constrained compared to the present fluid flow.

\section{Initial perturbation parameter}

The perturbations applied to the system should be small enough to prevent large unphysical divergence of the system. Typically large deviation of density can cause unphysical flow behavior. The perturbation should also be large enough to not be overwhelmed by round-off errors which cannot always be avoided. ${ }^{20}$

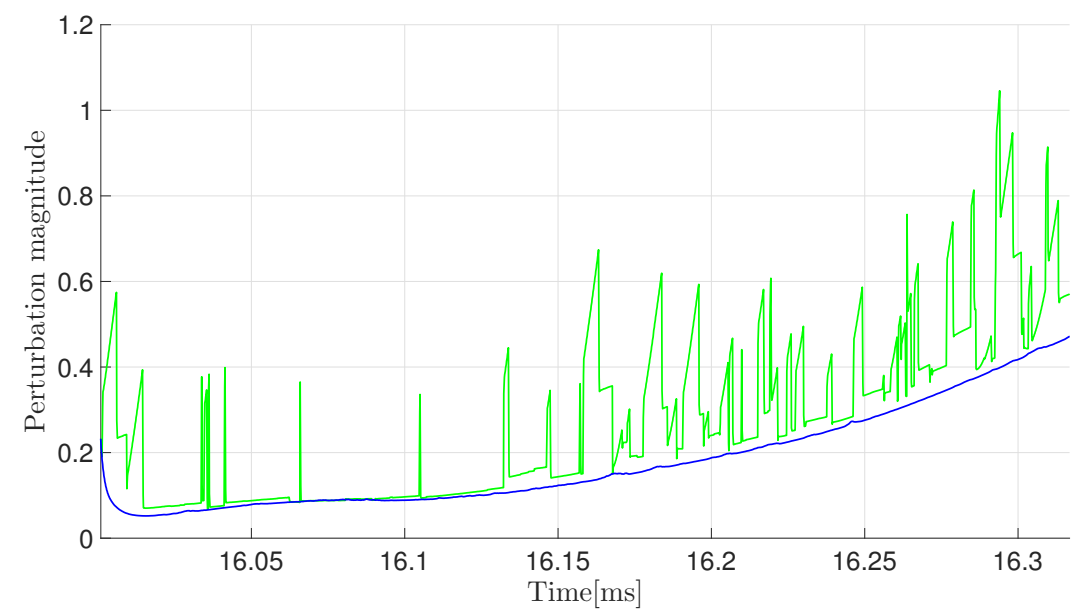

Figure 3: Time evolution of the perturbation $\mathrm{L}_{2}$-norm for progress variable using two different initial perturbation. Black line: initial perturbation of norm 1. Green line: initial perturbation of norm $1 e^{-4}$ multiplied by $1 e^{4}$ for plotting purposes.

In Fig. 3, the time evolution of a perturbation is plotted for two different initial perturbation magnitudes. For a perturbation of norm 1, the evolution in time is smoother than for the initial perturbation of norm $1 e^{-4}$. This difference can be attributed to the rise of round off errors for smaller perturbations. Indeed, for 
a number of grid points of the order $1 e^{6}$, the local perturbation is on average of the order of $1 e^{-10}$ when the perturbation norm is of the order of $1 e^{-4}$.

\section{Local Lyapunov exponent averaging time}

As for the averaging time (described as $s$ iteration in ${ }^{5}$ and above), Benettin et al. suggest that the critical parameter is not the local expansion rate averaging time but the total averaging time. However, to use effectively the Gram-Schmidt procedure, it is necessary to be able to discriminate between the vectors. For slowly growing vectors, a very small Lyapunov exponent averaging time can lead to mix the contribution of some pair of vectors as will be observed in Sec. B. For the sole purpose of Gram-Schmidt vector computations, a large averaging time does not cause problems when positive Lyapunov exponents are computed. For negative exponents, the perturbation component aligned with the corresponding Lyapunov vector can decrease in size until being overwhelmed by numerical errors.

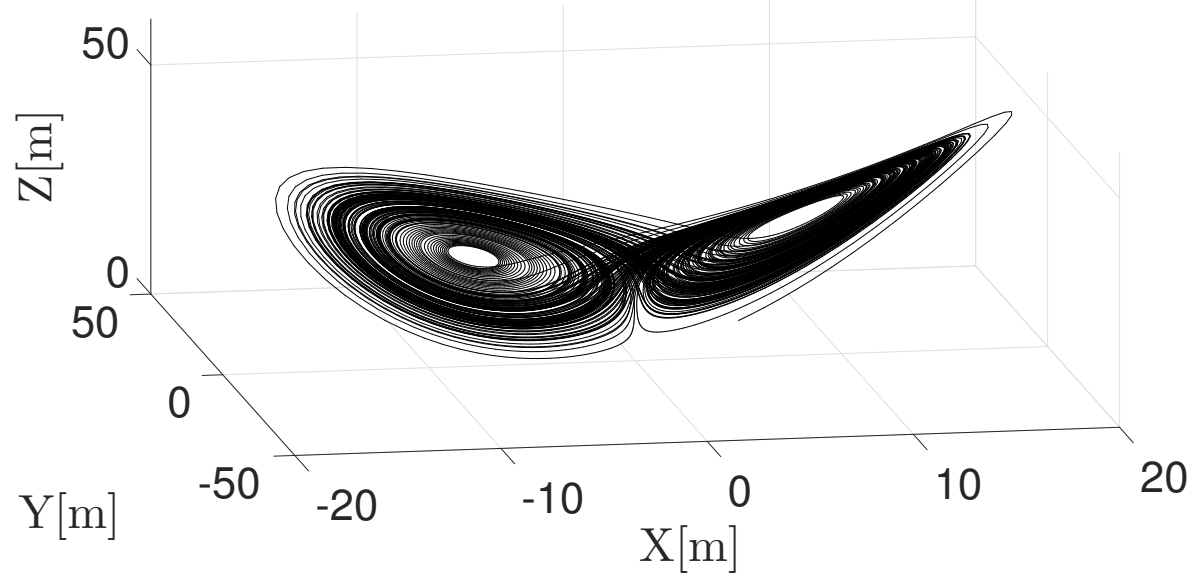

Figure 4: Visualization of the Lorenz attractor obtained from 1 realization with parameters $(\sigma, \rho, \beta)=$ $(10,28,8 / 3)$, and initial condition $(x, y, z)=(1,1,1)$

Since this kind of problem occurs for the last exponents, it is easier to observe in a simple system like the Lorenz attractor. For a particular and well-known set of parameters $(\sigma, \rho, \beta)=(10,28,8 / 3)$, the solution trajectory takes the form illustrated in Fig. 4. The state-space of the system is three-dimensional, and therefore, three Lyapunov exponents can be computed in this case. The third Lyapunov exponent is known to be $-14.5,{ }^{6}$ meaning that any perturbation aligned with the third Lyapunov vector shrinks at an exponential rate.

We use an initial 3D perturbation of $\mathrm{L}_{2}$-norm $2 e^{-10} \mathrm{~m}$ and integrate the governing equation for 20000 timesteps. We use an explicit $4^{t h}$ order Runge-Kutta timestepping with a timestep $\Delta t=0.01 \mathrm{~s}$. The transient time parameter mentioned above is not used (it is set to zero for now), so the procedure of Benettin et al. ${ }^{5}$ is followed to compute the Lyapunov exponents. The third computed exponent is shown in the Table 1 for different averaging times. As the averaging time increases, the initial perturbation shrinks below the numerical accuracy and results in a reduced absolute value of the exponent. Therefore, the upper limit of the averaging time is not only dictated by the alignment of the vectors in the rapidly growing direction, it should also be set to prevent shrinking vectors from disappearing. For the system analyzed in this paper, these considerations are not relevant since they would require to resolve all the exponents up to the negative ones. However it is important when considering the validation procedures for the solver.

\section{Transients dissipation time}

The third parameter that should be set is a transient time period during which no recording is done, right after every modification of the perturbation (every orthonormalization). While the need for such a transient time is debatable for weakly unconstrained system like Lorenz attractor or matricial cocycles, it is not possible to avoid for a CFD simulation. Every time the solution vector is artificially changed, there is no guarantee that the resulting field satisfies the basic conservation equation. In low-mach number solvers, this 
Table 1: Evolution of the third exponent value for the Lorenz attractor with increasing the averaging time

\begin{tabular}{||c||c||}
\hline Averaging time $[\mathrm{s}]$ & $\lambda_{3}$ \\
\hline \hline 0.6 & -14.5412 \\
\hline 1.2 & -10.4063 \\
\hline 2.4 & -5.2162 \\
\hline
\end{tabular}

is corrected immediately after one timestep but can result in oscillations especially if the pressure at the previous timestep is used as a guess for the new pressure at the next timestep (pressure is accumulated).

To remove transient effects, it is necessary to wait for some time before starting to record the Lyapunov exponents. In order to follow as closely as possible the procedure of Benettin et al., the perturbations are orthogonalized when the transient time ends for the sole purpose of recording without being modified in the actual simulations (modifying the simulations would again create the transients). Then the simulations are carried in the way described by Bennetin et al. ${ }^{5}$

The final procedure is laid out as illustrated in Fig. 5. The three parameters mentioned above are highlighted in the diagram by using bold font.

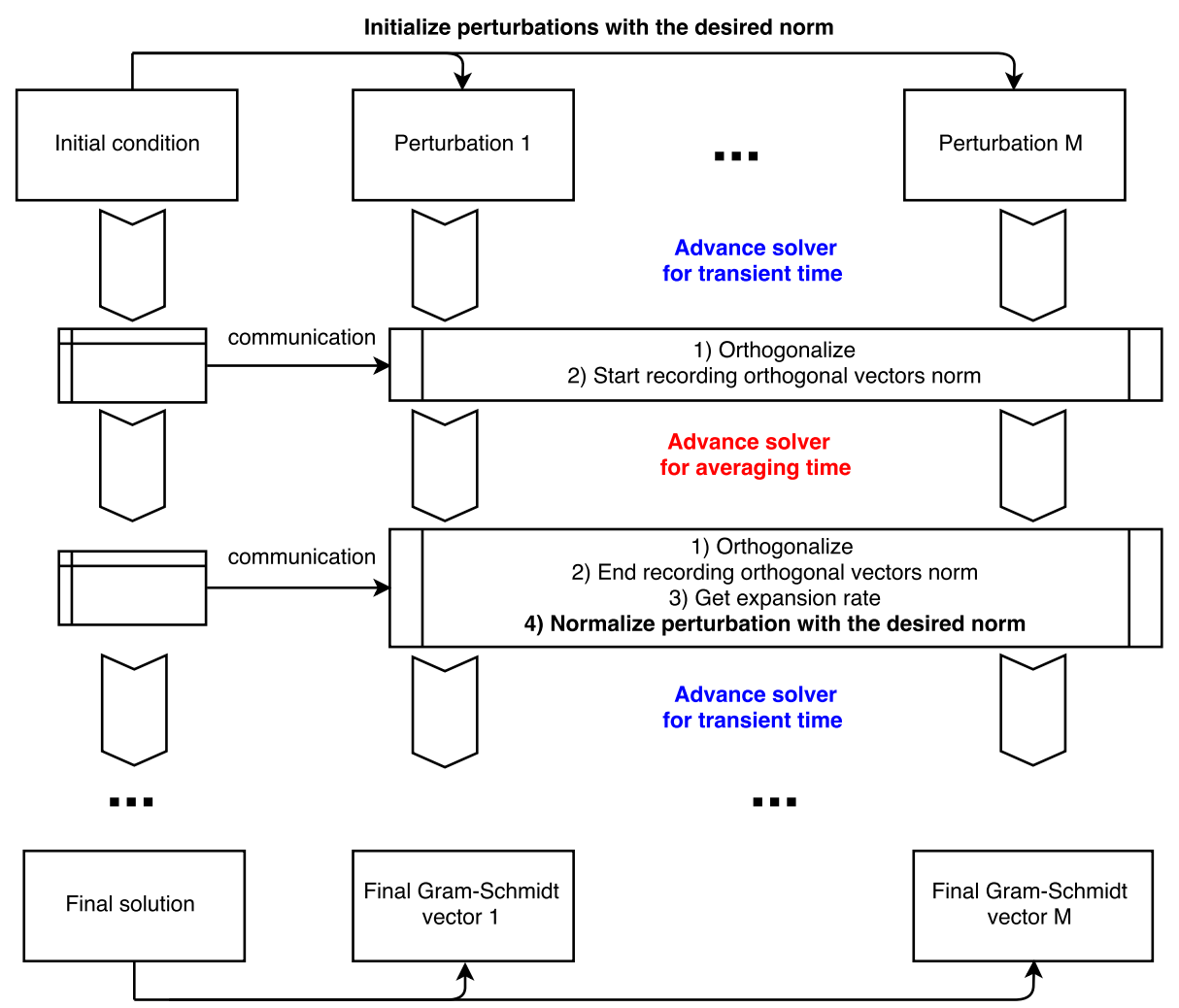

Figure 5: Diagram of the numerical procedure for computing $M$ Lyapunov exponents. The bold font shows where the parameters described in Sec. A are used. The bold black font denotes a usage of the initial perturbation norm. The bold blue font denotes the usage of the transient time. The bold red font denotes the usage of the local exponent averaging time.

In the actual implementation, several full-scale calculations are run simultaneously. This corresponds to the baseline reference solution and the $M$ perturbed simulations. At the end of the transient time or the averaging time, the independent simulations communicate with the reference simulation to determine the perturbation growth, and with other simulation for orthogonalization purposes. The code used here has been set up for parallel computations using MPI. An additional layer of processor coloring is added to mark 
which simulation each processor is responsible for. This way, the parallalelization structure of the original code can be kept as it was with little intrusion in the code. The orthonormalization and matrix storing are handled using the PETSc library ${ }^{21}$ and its extension, the SLEPc library. ${ }^{22}$ To minimize numerical error during the orthogonalization procedure the Modified Gram-Schmidt orthogonalization is used. ${ }^{23}$

\section{B. Choice of parameters}

With the numerical procedure presented above, three parameters need to be set: the transient time, the averaging time and perturbation norm. The goal is to determine a set of values that do not impact the computed exponent values adversely. As a test case, the first three exponents are computed using different parameters values and the same initial conditions.

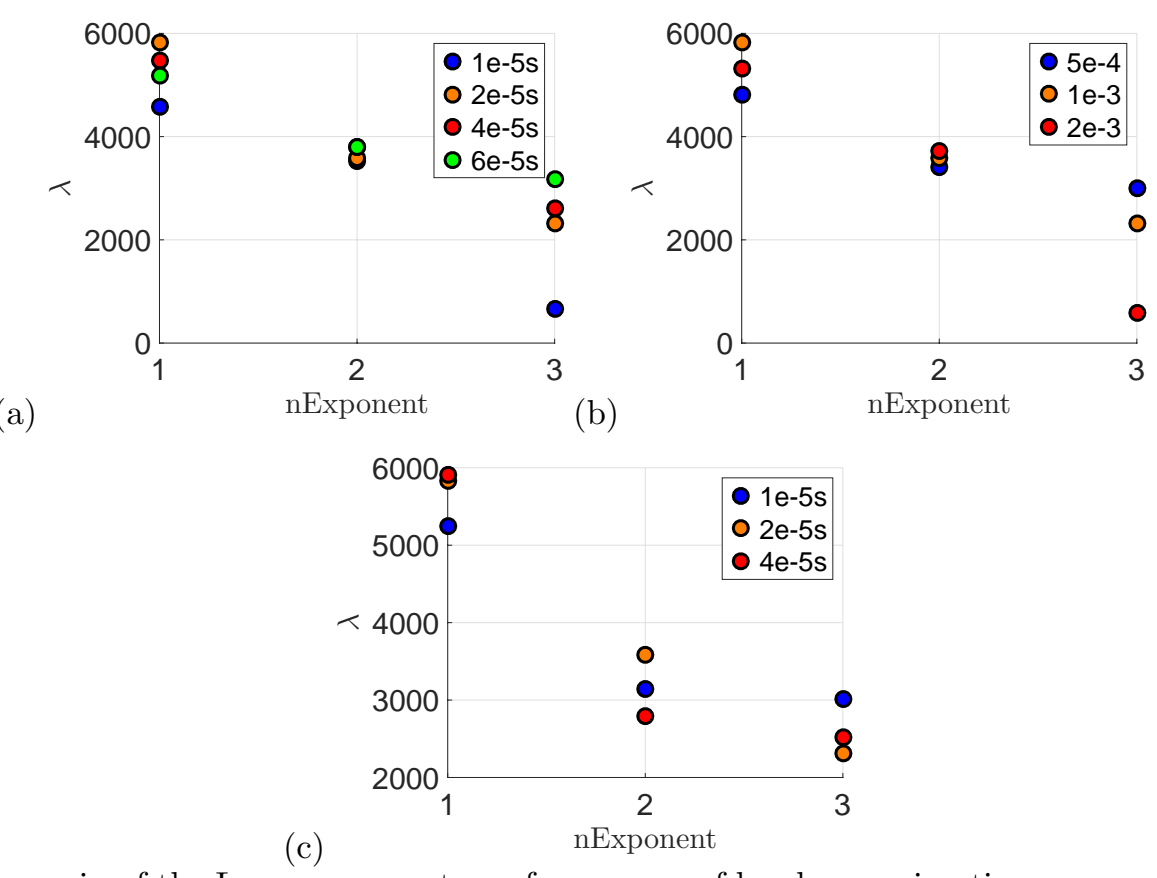

Figure 6: Differences in of the Lyapunov spectrum for a range of local averaging time parameters (a), initial perturbation norm parameters (b) and transient time parameter (c).

As can be seen in Fig 6, for the range of parameters inspected, the Lyapunov exponents do not appear to be impacted very much in the sense that the structure of the spectrum is similar with the range of values roughly identical. The averaging time and the perturbation magnitude seem to have the biggest impact at after the second exponent only, although the results do not seem to converge in a particular direction. It is interesting to note that the perturbation size has almost no impact on the first two computed exponents meaning that the expansion of the perturbation is almost exactly proportional to the initial perturbation size ruling out any effect of round-off errors evoked in Sec. A. As a result in the following computations, the transient time will be fixed at $2 e^{-5} s$, the perturbation norm will be fixed at $1 e^{-3}$ and the averaging time will be fixed at $2 e^{-5} s$.

\section{Safety checks during orthogonalization}

For very large simulations made of large number of exponents and large size for the state-vector, it is necessary to design the procedure in a resilient way. The most fragile part of the algorithm is the orthogonalization procedure which works only if the rank of the Gram-Schmidt vectors is found to be maximal by the numerical method. It is possible that in certain trajectories simulated containing the slowest growing vectors, the corresponding perturbations become linearly dependent with the rest of the perturbations. Each perturbation vector tends to be aligned with the fastest growing vector. Thus the slower growing vectors become more and more indistinguishable. In that case, a safety check should be implemented such that even if orthogonalizing the solution with the rest of the vectors leads to an error, the simulation can continue. Using the GrammSchmidt method, the vectors are treated one by one. Once a vector is found linearly dependent of the 
previous ones, the orthogonalization of this particular vector is skipped, and the values of its components are replaced by random numbers until the next orthogonalization takes place. During this process only the Lyapunov exponents associated with the vectors that did not fail are recorded. This orthogonalization process is illustrated in Fig. 7.

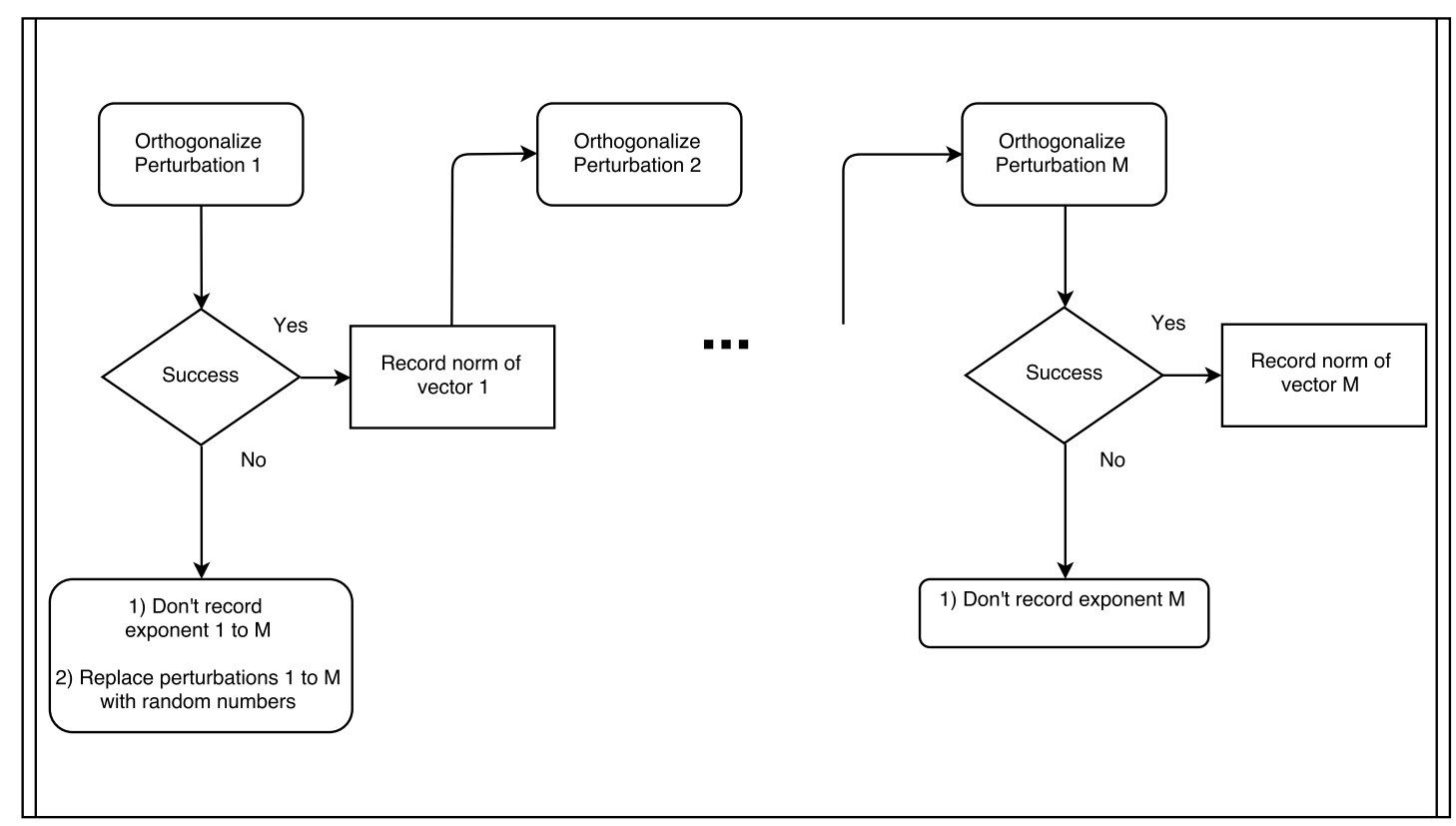

Figure 7: Diagram illustrating the resilient modification of the orthogonalization process in the case where a vector linearly dependent of the other ones is found.

\section{Reduction of state-space}

Before starting the investigation of a larger number of Lyapunov exponents, one can try to reduce the computational cost of the problem. One way to achieve this is by reducing the size of the state-vector. Not only is the inter-process communication cost lower at each step, but the orthogonalization itself is more robust. In this section, we look for relations between state-vector fields that could lead to the reduction of state-space.

\section{Relation mass flux-velocity}

The mass flux $\{\rho U, \rho V, \rho W\}$, are provided along with $\{U, V, W\}$ in the state vector. In NGA, the mass fluxes are obtained by multiplying the velocity with the density at mid time-step. The three mass fluxes can therefore be replaced by the mid time-step density $\rho_{\text {mid }}$. To make sure that the impact of this modification on the Lyapunov spectrum is indeed minimal, the expansion rate $\Lambda_{i}$ is treated as a random variable which is sampled at each recording. Using the parameters set in Sec. B, one exponent is computed. The PDF of the expansion rate can then be drawn.

As can be observed in Fig. 8, not only is the mean value of the expansion rate is similar but the entire PDF matches between a case with mass fluxes included and mid-time density included. The $\mathrm{L}_{2}$ norm of both PDF is below $5 \%$ of the total norm. This result gives strong confidence in the equivalence between the state-vectors.

\section{The role of pressure}

In low-Mach number solvers, the pressure field is used to enforce mass conservation. Algorithmically, the pressure field is a measure of the level of lack of mass conservation. In NGA, because the first pressure gradient used in the first outer-iteration of the timestep is obtained from the previous timestep, it was 


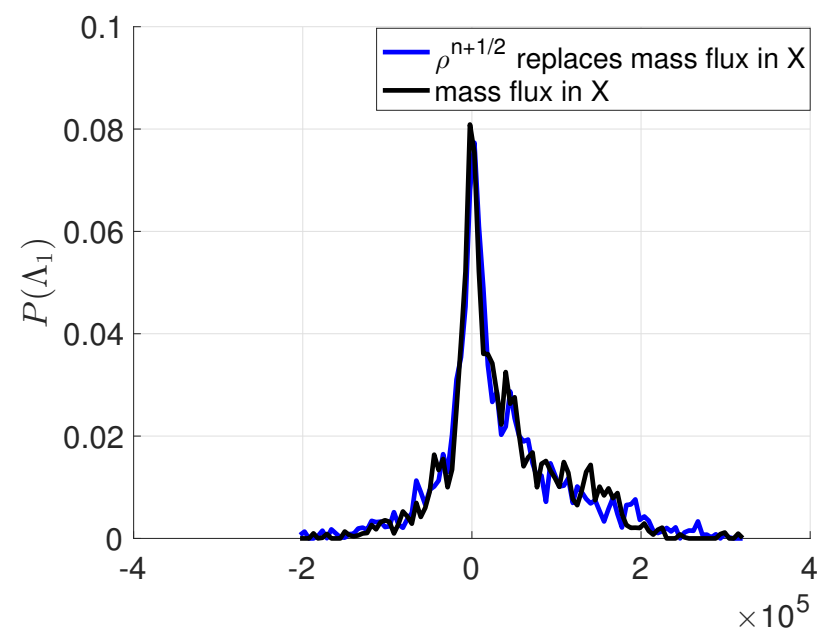

Figure 8: PDF of the first expansion rate considering a state-vector including the 3 mass fluxes (black line) and the state-vector including the mid-time density (blue line)

necessary to include it in the state-vector. However, it could be argued that due to the iterative procedure in this algorithm, the first guess of pressure gradient has little effect on the final result, especially for explicit methods like the one used. Two cases are run: 1$)$ with a cancellation of the initial pressure gradient $\left((\text { Press })_{3}^{n}\right.$ in Eq. 10 is replaced by a null vector) and a complete removal of pressure from the state vector, 2) with simply removing pressure from the state vector while keeping (Press ${ }_{3}^{n}$ as first guess in the semi-implicit procedure. When the value of pressure is used in the state-vector, its magnitude is shifted at every location so that the first cell in the $\mathrm{x}, \mathrm{y}$ and $\mathrm{z}$ direction match (only the pressure gradient is meaningful in a low-Mach number solver).

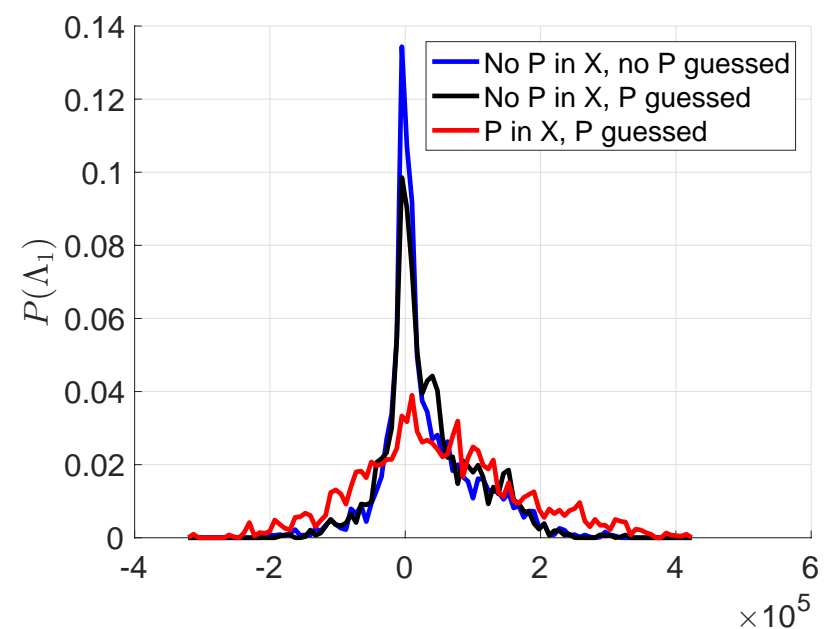

Figure 9: PDF of the first expansion rate considering a state-vector without pressure contribution and without changing the low-Mach procedure (black line), without pressure contribution and changing the low-Mach procedure (blue line), with pressure contribution (red line)

In Fig. 9, it can be observed that neglecting the contribution of pressure to the state-vector approximately or rightfully, result in similar PDF for the first expansion rates. The $\mathrm{L}_{2}$ norm of both PDF is below $6 \%$ of the total norm. However, once the pressure gradient is taken into account, although the mean value is similar to the previous computations, the PDF is flatter which reduces the convergence speed of the procedure. The contribution of pressure in the state vector overwhelms the rest of the fields which is non-desirable for the current purpose. Therefore, in the following computation, the pressure field is simply removed from the state-vector while it is still being accumulated in the low-Mach procedure. Fig. 9 provides support that it will not affect the conclusions regarding the Lyapunov spectrum. 


\section{Lyapunov spectrum of turbulent combustion}

Using the algorithm detailed in Sec. IV along with the approximations justified above, it becomes possible to compute large amount of exponents for the turbulent combustion configuration. In this work, the focus is only limited to the structure of the spectrum with the perspective of inferring the dimensionality of the attractor using Eq. 6. The first 99 exponents are computed using 32 processors per simulation for a total computational time of $50 \mathrm{~K}$ processor hours.

As mentioned in Sec. IV, the Lyapunov exponents are time dependent because they are phase-space dependent quantities. The $\lambda_{i}$ can be obtained from the time averaging of the $\Lambda_{i}$. To show convergence of the Lyapunov exponents, the running averages are plotted in Fig. 10. It is interesting to note that the convergence rate scales with the mean value of the exponent meaning that the standard deviation of the $\mathrm{PDF}$ of the expansion rate scales also with the mean value. As a result, the PDF of the expansion rates would have similar shapes across different Lyapunov exponents.

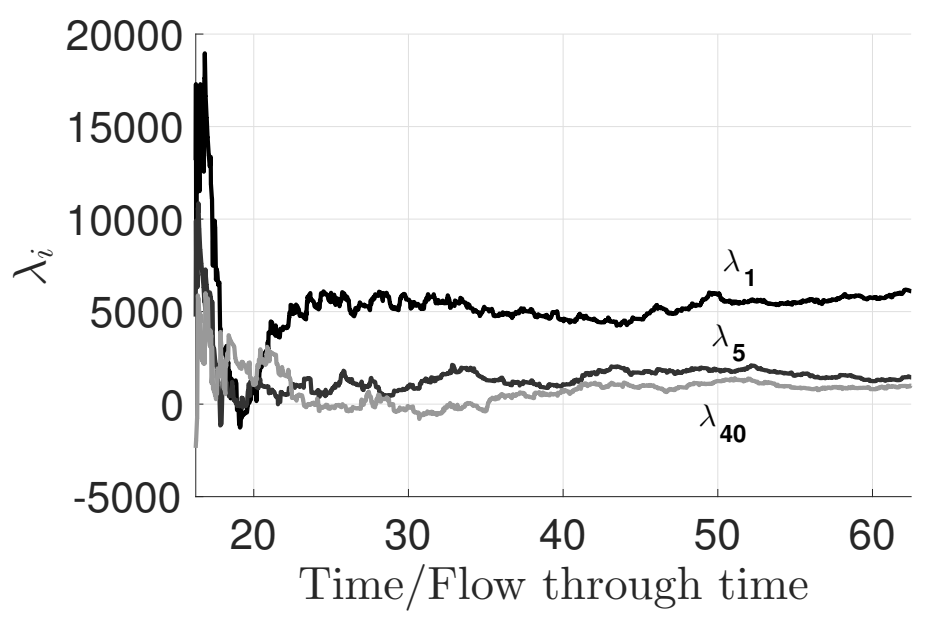

Figure 10: Running average of expansion rates 1, 5, 40. The lighter color, the larger the index of the expansion rate.

The resulting spectrum of Lyapunov exponents is plotted for both resolutions in Fig. 11. First, it can be observed that the first exponent exhibits a large value while the following values quickly decay and stabilize at values slightly larger than zero. This structure follows the convex shape of the spectrum shown by previous studies for turbulent flow, ${ }^{6,11}$ however the large value of the first exponent appears as a singular feature. Second, the slow decay of the spectrum to zero and the large first exponent also suggest that finding the dimension of the attractor could require the computation of many more exponents. Finally the spectrum appears scattered, which could be due to a lack of convergence of the running averages, or to the quality of the orthogonalization.

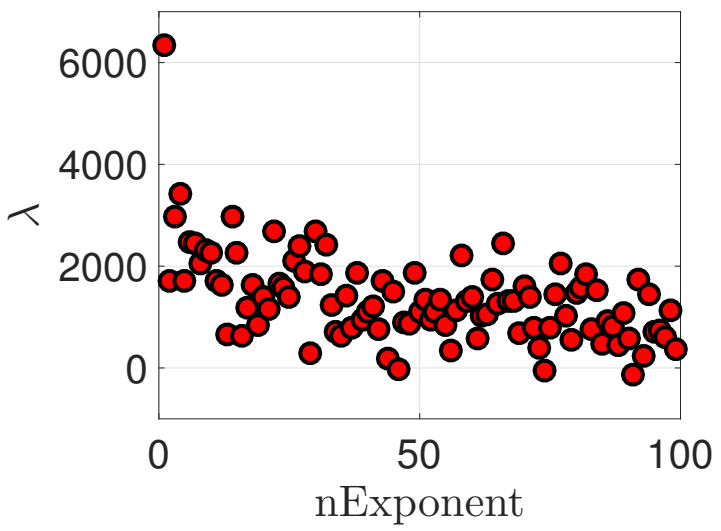

Figure 11: Lyapunov exponents for a fine $(128 \times 64 \times 64$, in green $)$ and a coarse simulation $(64 \times 64 \times 64$, in blue). 
On the path of increasing the number of computed exponents, it is important to recognize that the main numerical issue of the algorithm resides in the orthogonalization procedure. The Gram-Schmidt orthogonalization uses a Q-R decomposition of the perturbation matrix containing the solution vectors. This matrix has a size $\mathcal{D} \times M$ where $\mathcal{D}$ is the size of each solution vector and $M$ is the number of exponents to compute. The procedure can lead to issues if the matrix is badly conditioned. ${ }^{23}$ In Fig. 12, the condition number of a matrix (defined as the ratio of the largest to the lowest singular value) is plotted against the number of exponents computed. As can be observed, the more exponents are needed, the more difficult the procedure is numerically. This finding suggests that further improvement can be introduced in the method through more efficient orthogonalization algorithm or matrix preconditioning.

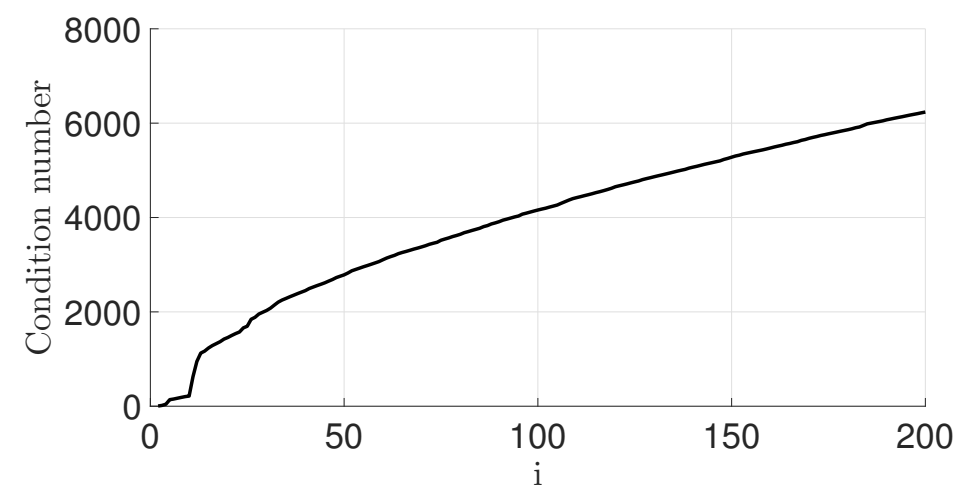

Figure 12: Evolution of the condition number of the matrix where solution vectors are orthogonalized with the number of solution vectors.

\section{Conclusions and perspective}

Numerical implementation and choices for the computation of Lyapunov exponent in a high dimensional system have been described. It is highlighted that three parameters (transient time, averaging time, perturbation magnitude) need to be carefully set in order to perform such computations. Additionally the state-space of the system should be appropriately defined in order to use the dynamical system theory. The computation of the first 99 Lyapunov exponents is performed for a canonical turbulent combustion system. The results show similar convex shape as previously studied turbulent Poiseuille flows. However, conversely to these configurations, the first exponent appears to be remarkably larger, while the rest of the spectrum slowly decays to zero. Therefore the first 99 exponents are not enough to determine the attractor dimension of the system which would require a larger simulation. The feasibility of such a simulation would however require to alleviate numerical issues in the orthogonalization procedure.

\section{Acknowledgements}

This work was financially supported by an AFOSR research grant (FA9550-15-1-0378) with Dr. Chiping $\mathrm{Li}$ as program manager. The authors thank NASA HECC for generous allocation of computing time on NASA Pleiades machine. 


\section{References}

${ }^{1}$ Kolmogorov, A. N., "A refinement of previous hypotheses concerning the local structure of turbulence in a viscous incompressible fluid at high Reynolds number," Journal of Fluid Mechanics, Vol. 13, 1962, pp. 82-85.

-2Kraichnan, R., "On Kolmogorov's inertial-range theories," Journal of Fluid Mechanics, Vol. 62, 1974, pp. 305-330.

>3Pitsch, H., "Large-eddy simulation of turbulent combustion," Annual Review of Fluid Mechanics, Vol. 38, 2006, pp. 453482.

${ }^{4}$ Lietz, C., Hassanaly, M., Raman, V., Kolla, H., Chen, J., and Gruber, A., "Large Eddy Simulation of Premixed Flame Flashback in a Turbulent Channel," 52nd AIAA Aerospace Science Meeting and Exhibit, 2014.

${ }^{5}$ Benettin, G., Galgani, L., Giorgilli, A., and Strelcyn, J.-M., "Lyapunov characteristic exponents for smooth dynamical systems and for Hamiltonian systems; A method for computing all of them. Part 2: Numerical application," Meccanica, Vol. 15, 1980, pp. 21-30.

${ }^{6}$ Keefe, L., Moin, P., and Kim, J., "The dimension of attractors underlying periodic turbulent Poiseuille flow," Journal of Fluid Mechanics, Vol. 242, 1992, pp. 1-29.

${ }^{7}$ Eckmann, J.-P. and Ruelle, D., "Ergodic-Theory of Chaos and Strange Attractors," Review of Modern Physics, Vol. 57, 1985, pp. 617-656.

${ }^{8}$ Grappin, R. and Léorat, J., "Lyapunov exponents and the dimension of periodic incompressible Navier-Stokes flows: numerical measurements," Journal of Fluid Mechanics, Vol. 222, 1991, pp. 61-94.

${ }^{9}$ Constantin, P., Foias, C., Manley, O. P., and Temam, R., "Determining modes and fractal dimension of turbulent flows," Journal of Fluid Mechanics, Vol. 150, 1985, pp. 427-440.

${ }^{10}$ Vastano, J. A. and Moser, R. D., "Short-time Lyapunov exponent analysis and the transition to chaos in Taylor-Couette flow," Journal of Fluid Mechanics, Vol. 233, 1991, pp. 83-118.

11 Inubushi, M., Takehiro, S., and Yamada, M., "Regeneration cycle and the covariant Lyapunov vectors in a minimal wall turbulence," Physical Review E, Vol. 92, 2015, pp. 023022.

${ }^{12}$ Patil, D. J., Hunt, B. R., Kalnay, E., Yorke, J. A., and Ott, E., "Local Low Dimensionality of Atmospheric Dynamics," Physical Review Letters, Vol. 86, 2001, pp. 5878-5881.

$\checkmark{ }^{13}$ Benettin, G., Galgani, L., Giorgilli, A., and Strelcyn, J.-M., "Lyapunov characteristic exponents for smooth dynamical systems; A method for computing all of them. Part 1: Theory," Meccanica, Vol. 15, 1980, pp. 21-30.

${ }^{14}$ Frederickson, P., Kaplan, J. L., Yorke, E. D., and Yorke, J. A., "The Lyapunov dimension of strange attractors," Journal of Differential Equations, Vol. 49, 1983, pp. 185-207.

${ }^{15}$ Pitsch, H., "A C++ Computer Program for 0-d and 1-D Laminar Flame Calculations," RWTH Aachen.

$\checkmark{ }^{16}$ Rosales, C. and Meneveau, C., "Linear forcing in numerical simulations of isotropic turbulence: Physical space implementations and convergence properties," Physics of Fluids, Vol. 17, 2005.

${ }^{17}$ Bell, J. B., Day, M. S., Grcar, J. F., and Lijewski, M. J., "Active control for statistically stationary turbulent premixed flame simulations," Communications in Applied Mathematics and Computational Science, Vol. 1, 2006, pp. $29-51$.

${ }^{18}$ Hassanaly, M., Raman, V., Koo, H., and Colkett, M. B., "Influence of Fuel Stratification on Turbulent Flame Propagation," 53rd AIAA Aerospace Science Meeting and Exhibit, 2015.

$\checkmark{ }^{19}$ Desjardins, O., Blanquart, G., Balarac, G., and Pitsch, H., "High order conservative finite difference scheme for variable density low Mach number turbulent flows," Journal of Computational Physics, Vol. 227, No. 15, JUL 20 2008, pp. 7125-7159.

${ }^{20}$ Senoner, J.-M., García, M., Mendez, S., Staffelbach, G., Vermorel, O., and Poinsot, T., "Growth of Rounding Errors and Repetitivity of Large Eddy Simulations," AIAA Journal, Vol. 46, 2008, pp. 1773-1781.

${ }^{21}$ Balay, S., Brown, J., Buschelman, K., Eijkhout, V., Gropp, W. D., Kaushik, D., Knepley, M. G., McInnes, L., Smith, B. F., and Zhang, H., "PETSc Users Manual," Argonne National Laboratory, Tech. Rep., 2013.

${ }^{22}$ Hernandéz, V., Román, J. E., and Vidal, V., "SLEPc: A scalable and flexible toolkit for the solution of eigenvalue problems," ACM Trans. Math. Software, Vol. 31, 2005, pp. 351-362.

${ }^{23}$ Hernandéz, V., Román, J. E., Tomás, and Vidal, V., "Orthogonalization Routines in SLEPc," Tech. rep., Universidad Politécnica de Valencia, 2007. 